\title{
Efecto de la adición del rotífero Brachionus rotundiformis (Tschugunoff, 1921) sobre la calidad del agua y la producción, en cultivos super-intensivos de camarón blanco del Pacífico Litopenaeus vannamei (Boone, 1931)
}

\author{
Effect of the rotifer Brachionus rotundiformis (Tschugunoff, 1921) addition on water quality \\ and production, in super-intensive cultures of the Pacific white shrimp \\ Litopenaeus vannamei (Boone, 1931)
Alfredo Campaña-Torres ${ }^{1}$, Luis R. Martínez-Córdova ${ }^{2 *}$, Humberto Villarreal-Colmenares ${ }^{1}$, Jorge Hernández-López ${ }^{1}$, Josafat M. Ezquerra-Brauer ${ }^{3}$ y Edilmar Cortés-Jacinto ${ }^{1}$ \\ ${ }^{1}$ Centro de Investigaciones Biológicas del Noroeste, Apdo. Postal 128, La Paz, BCS 23090, México \\ ${ }^{2}$ Departamento de Investigaciones Científicas y Tecnológicas, Universidad de Sonora, \\ Apdo. Postal 1819, Hermosillo, Sonora, México \\ ${ }^{3}$ Departamento de Investigación y Posgrado en Alimentos, Universidad de Sonora, \\ Apdo. Postal 1658, C. P. 8300, Hermosillo, Sonora, México \\ *Imtz@guaymas.uson.mx
}

\begin{abstract}
The effectiveness of the rotifer Brachionus rotundiformis as exogenous natural feed combined with balanced feed on the production parameters of Litopenaeus vannamei and the water quality was evaluated. Different concentrations of rotifers were tested $(0,5,10,15$ and 20 rotifers $\mathrm{mL}^{-1}$ ) during 45 days. No significant differences were assessed in the phosphate levels among treatments. The $\mathrm{pH}$ level was statistically lower in the treatments with 15 and 20 rotifers $\mathrm{mL}^{-1}$. Nitrate, nitrite and ammonia were significantly lower in the treatments with low concentration of rotifers $\left(0\right.$ and $\left.5 \mathrm{~mL}^{-1}\right)$, while the highest values were achieved in the higher concentrations (15 and $20 \mathrm{~mL}^{-1}$ ) without reaching toxic levels. The weight gain, biomass, survival and specific growth rate of L. vannamei were significantly higher in the treatments with the higher number of rotifers (15 and $20 \mathrm{~mL}^{-1}$ ), whereas the lowest values were found in the control treatment $\left(0 \mathrm{~mL}^{-1}\right)$. The feed conversion ratio (FCR) was statistically lower in the treatment with the highest concentration of rotifers, while the highest FCR was found in the control. It is concluded that the use of the rotifer $B$. rotundiformis in high concentrations improves the production parameters on the Pacific white shrimp, without deteriorating the water quality.
\end{abstract}

Key words: Production parameters, natural exogenous food, shrimp feeding, shrimp nutrition
Resumen.- Se evaluó la efectividad del rotífero Brachionus rotundiformis como alimento natural exógeno combinado con alimento balanceado sobre los parámetros de producción de Litopenaeus vannamei y la calidad del agua. Se probaron diferentes concentraciones de rotíferos: $0,5,10,15$ y 20 rotíferos $\mathrm{mL}^{-1}$ durante 45 días. No se encontraron diferencias en los niveles de fosfatos entre los tratamientos. El pH fue estadísticamente menor en los tratamientos con 15 y 20 rotíferos $\mathrm{mL}^{-1}$. Los nitratos, nitritos y amonio fueron significativamente menores en los tratamientos con baja concentración de rotíferos ( 0 y $5 \mathrm{~mL}^{-1}$ ), mientras que los valores más elevados se presentaron en las concentraciones más altas (15 y $\left.20 \mathrm{~mL}^{-1}\right)$ sin que estas llegaran a ser tóxicas. El peso ganado, biomasa, supervivencia y tasa de crecimiento específico de L. vannamei fueron significativamente mayores en los tratamientos con mayor número de rotíferos (15 y $20 \mathrm{~mL}^{-1}$ ), mientras que los valores más bajos se encontraron en el tratamiento control (0 $\mathrm{mL}^{-1}$ ). El factor de conversión alimenticia (FCA) fue estadísticamente menor en el tratamiento con mayor concentración de rotíferos, mientras que el factor de conversión alimenticia (FCA) más alto se encontró en el tratamiento control. Se concluye que el uso del rotífero B. rotundiformis en altas concentraciones mejora los parámetros de producción del camarón blanco del Pacífico sin llegar a deteriorar de manera importante la calidad del agua.

Palabras clave: Parámetros de producción, alimento natural exógeno, alimentación de camarón, nutrición de camarón 


\section{Introducción}

La acuacultura es una de las actividades económicas de mayor desarrollo en el mundo lo cual se ve reflejado en volúmenes de producción superiores a los 60 millones de toneladas (FAO 2006). En México, esta tendencia se ha dado especialmente en una de sus ramas que es la camaronicultura, con una producción anual de alrededor de 95.000 toneladas en el 2006 (Gutierrez-Venegas 2006).

Existen muchos aspectos que deben abordarse para que la camaronicultura siga expandiéndose tanto en México como a nivel mundial, destacándose el manejo adecuado de los sistemas de cultivo, orientado sobre todo a una actividad sustentable. Esto implica la necesidad de manejar los sistemas de producción de tal forma que la actividad sea económicamente rentable y ecológicamente responsable, es decir, que la producción sea efectiva sin dañar el ambiente. Dos aspectos que pueden contribuir a lo anterior son el manejo adecuado del alimento balanceado y estrategias de alimentación que tomen en cuenta la productividad natural (Tacon 2002).

Se considera que el insumo más costoso en la producción comercial de camarón es el alimento artificial, el cual llega a representar más del 50\% de los costos operativos en granjas camaronícolas semiintensivas (Zendejas-Hernández 1994, Martínez-Córdova et al. 2003, Pontes et al. 2006). Los alimentos artificiales comerciales que se utilizan en la actualidad para el cultivo de camarón se formulan bajo condiciones de laboratorio con lo cual se ignora la contribución del alimento natural y el efecto de las condiciones de cultivo (Jory 2000, Tacon 2002).

Diversos autores han documentado la importancia del alimento natural en la nutrición del camarón cultivado, demostrando que puede aportar hasta más del 70\% de su requerimiento (Jory 2000, Martínez-Córdova et al. 1998, Enríquez 2003, Martínez-Córdova \& Enríquez-Ocaña 2007). Se ha encontrado que los principales elementos de la productividad natural a partir de los cuales el camarón se alimenta, son organismos del zooplancton y del bentos (Chiu \& Chen 1992, Barraza-Guardado 1996, Peña Messina 1999, Martinez-Cordova \& Peña-Messina 2005). A pesar de este conocimiento, es notable que hasta ahora no se hayan obtenido grandes ventajas de ello en las granjas comerciales. El problema radica en la dificultad de mantener una adecuada biomasa de estos organismos durante todo el cultivo, de tal manera que puedan representar una contribución significativa en la nutrición del camarón (White 1986, Barraza-Guardado 1996, Martínez Córdova et al. 1998).

Se han utilizado diversas prácticas para incentivar el desarrollo de la productividad natural en los estanques de cultivo como lo son: la fertilización orgánica e inorgánica (Jory 1996, De la Lanza \& Hernández 1998), el uso de sustratos fermentados (alfalfa seca mezclada con aceite de bacalao, vitamina C, melaza y agua) (Martínez-Córdova et al. 2002), instalación de sustratos artificiales para promover películas bacterianas (Martínez-Córdova et al. 2004, Arnold et al. 2006, Ballester et al. 2007), uso de bio-flocs bacterianos (De Schryver et al. 2008), y hasta la incorporación de alimento natural exógeno a los sistemas (Molina-Poveda et al. 2006).

El hecho de que los camarones cultivados puedan aprovechar el alimento natural generado en los estanques (fitoplancton, zooplancton y bentos) puede representar una reducción significativa en los gastos operativos de una granja. Además, se ha demostrado que este alimento natural posee un alto valor nutricional (McKinnon et al. 2003, Palmtag et al. 2006); por ejemplo, las proteínas del zooplancton poseen una mayor digestibilidad que las contenidas en alimentos artificiales a base de harina de pescado, calamar, etc. (Castille \& Lawrence 1989, Martínez-Córdova et al. 1999, 2002, Cortés-Jacinto et al. 2003). Por otra parte, el uso de mayor cantidad de alimento natural y la reducción en la administración de alimento artificial eventualmente reduciría el impacto ambiental provocado por las descargas acuícolas (Tacon 2002).

El uso de rotíferos como alimento natural, se ha implementado en el cultivo de diversas especies acuáticas, incluyendo camarones (Fengqi 1996, Wilcox et al. 2006). No obstante, la administración de rotíferos y otros organismos (copépodos y artemia), se lleva a cabo solamente durante el desarrollo larval de los camarones (Cabrera et al. 2002, De Lima \& Souza-Santos 2007) y no es común en la engorda. Es por ello que no existen estudios que hayan evaluado el uso de rotíferos como alimento en la engorda de camarones peneidos ni su efecto sobre la calidad del agua.

El objetivo del presente estudio fue evaluar el uso del rotífero Brachionus rotundiformis (Tschugunoff, 1921) como alimento natural exógeno a cuatro diferentes concentraciones, sobre la respuesta productiva de los juveniles de camarón blanco Litopenaeus vannamei (Boone, 1931) bajo condiciones de cultivo súperintensivo, así como en la calidad en agua del sistema.

\section{Material y métodos}

El estudio se desarrolló en instalaciones de la Unidad Experimental Kino (UEK) del Departamento de Investigaciones Científicas y Tecnológicas de la 
Universidad de Sonora (DICTUS), ubicado en Bahía de Kino Sonora, México.

\section{Cultivo de microalgas y rotíferos}

Se realizó un cultivo de la microalga Nannochloropsis oculata utilizando el medio F/2 de Guillard (Guillard 1975). Las microalgas se cosecharon cuando alcanzaron una concentración promedio de $10 \pm 3 \times 10^{6}$ células $\mathrm{mL}^{-1}$, mientras se encontraban en crecimiento exponencial y se adicionaron al cultivo de rotíferos.

El mantenimiento de la cepa y el cultivo de Brachionus rotundiformis se realizó siguiendo la metodología descrita por Fukusho (1989) y Fengqi (1996). La cepa se obtuvo del Centro de Desarrollo Tecnológico de Especies Marinas (CEDETEM), ubicado en Tomatlán, Jalisco y se mantuvo en matraces Erlenmeyer de $2 \mathrm{~L}$ a una temperatura de $26 \pm$ $1^{\circ} \mathrm{C}$, a una densidad no mayor de $200 \pm 20$ rotíferos $\mathrm{mL}^{-1}$. En el caso de los cultivos masivos, éstos se realizaron primeramente en el laboratorio en garrafones de $20 \mathrm{~L} \mathrm{y}$ posteriormente se pasaron columnas de fibra de vidrio de $100 \mathrm{~L}$ colocadas dentro de un invernadero. Las condiciones de cultivo fueron: iluminación constante $\left(24 \cdot\right.$ día $\left.^{-1}\right)$ y temperatura ambiente promedio de $30 \pm 2^{\circ} \mathrm{C}$. Su cosecha se realizó cuando alcanzaron una densidad de 300 organismos $\mathrm{mL}^{-1}$.

\section{Bioensayo para la evaluación del rotífero como alimento natural exógeno}

El bioensayo tuvo una duración de 45 días, utilizando un diseño experimental simple en un arreglo completamente al azar con tres repeticiones por tratamiento. Las unidades experimentales consistieron en tanques de plástico con capacidad de 50 L. Se utilizó agua de mar filtrada (5 $\mu \mathrm{m})$ y esterilizada.

Los organismos experimentales fueron camarones de $0,2 \pm 0,01 \mathrm{~g}$. La talla de los organismos se estableció en función de que fuese posible mantenerlos en densidades súper-intensivas, ya que en esta talla todavía es posible mantener a los camarones en instalaciones cerradas (invernaderos, 'raceways', tanques súper-intensivos) si el clima aún no es favorable para su siembra.

En cada unidad experimental se colocaron 22 camarones (equivalente a $122 \mathrm{~m}^{-2}$ ). Se evaluaron cuatro concentraciones (tratamientos) de rotíferos: 0 (control), 5, 10, 15 y 20 organismos $\mathrm{mL}^{-1}$ en combinación con alimento artificial comercial. Los rotíferos se adicionaron una vez al día. El alimento artificial empleado fue Camaronina de Purina ${ }^{\circledR}$ (Agribran Purina ${ }^{\circledR}$, Ciudad Obregón, Sonora, México) y se proporcionó a razón del $5 \%$ de la biomasa del camarón, en dos raciones diarias (09:00 y 20:00 h). Cada día se retiraron restos de exoesqueleto producto de la ecdisis, camarones muertos, restos de alimento y heces fecales mediante sifoneo manual con una manguera de 1/8"; mediante este último proceso se recambió un volumen de agua del 5\% diario. Adicionalmente se llevó a cabo un recambio semanal del $50 \%$.

El número de rotíferos se contó diariamente con la finalidad de agregar solo la diferencia para completar la concentración de cada tratamiento. El conteo se realizó tomando cinco muestras de $1 \mathrm{~mL}$ de agua de cada unidad experimental, a las cuales se les agregó 1 $\mathrm{mL}$ de fijador (formol al 10\%); posteriormente se colocó la muestra de $1 \mathrm{~mL}$ en una cámara Sedgwick Rafter $^{\circledR}$ y fue analizada y contada con ayuda de un microscopio compuesto (Ward's ${ }^{\circledR}$ ); el total se multiplicó por 2 (factor de dilución).

El total del consumo diario de rotíferos se registró para el cálculo real del factor de conversión alimenticia (FCA).

\section{Mantenimiento y monitoreo de variables de calidad del agua}

Se mantuvieron niveles de oxígeno disuelto superiores a $5 \mathrm{mg} \mathrm{L}^{-1}$ mediante un soplador de 2 HP. Diariamente (7:00 y 14:00 h) se registraron la temperatura, el oxígeno disuelto, el pH y la salinidad, utilizando una sonda multi parámetros YSI 6600 (YSI Inc., Yellowspring, OH, USA). Semanalmente se midieron las concentraciones de nitrógeno amoniacal total (NAT), nitritos, nitratos y fosfatos con un espectrofotómetro HACH DR $4000^{\circledR} \mathrm{y}$ su respectivo kit de reactivos (Hach Co. Loveland, CO, USA), siguiendo las técnicas descritas en el manual del equipo; se tomaron cinco muestras de cada tanque.

\section{Evaluación de los parámetros de producción}

Semanalmente se realizaron biometrías de crecimiento, pesando individualmente cada uno de los camarones de cada unidad experimental para determinar el crecimiento semanal y la biomasa presente. La biomasa calculada se utilizó para estimar la cantidad de alimento a suministrar durante la semana siguiente. También se contaron los camarones vivos de cada tanque para determinar la supervivencia semanal.

Al final del experimento se contó y pesó cada uno de los camarones de cada tratamiento y se evaluó el desempeño de los organismos en términos del crecimiento final (CF), tasa de crecimiento específica (TCE), supervivencia (SF), factor de conversión alimenticia (FCA) y biomasa final (BF). 


\section{Análisis estadístico}

Los datos de la calidad de agua fueron analizados para determinar si tenían o no una disribución normal (prueba de Lilliefors) y si sus varianzas eran o no homogéneas (prueba de Barttlet); como cumplieron ambos supuestos, se les realizó un análisis de varianza (ANOVA) paramétrica de medidas repetidas y una prueba $a$ posteriori de comparación de medias (Tukey) (Conover 1980, Sokal y Rohlf 1979).

Los datos obtenidos de los parámetros de producción (talla final, supervivencia, biomasa final y FCA) fueron sometidos a un análisis exploratorio para determinar si eran o no normales y homocedásticos; como no cumplieron con alguna de las pruebas anteriores, se les aplicó un ANOVA no paramétrica (Kruskal-Wallis). Todas las pruebas se realizaron con un nivel de confianza del 95\%, utilizando el software estadístico Statgraphic Plus ${ }^{\circledR}$ 5.1. Los resultados de calidad del agua así como los de producción, se muestran como medias \pm desviación estándar.

\section{Resultados}

No se encontraron diferencias significativas entre los valores medios de temperatura, oxígeno disuelto y salinidad. Los valores de $\mathrm{pH}$ resultaron significativamente menores en los tratamientos con 15 y 20 rotíferos $\mathrm{mL}^{-1}$ en comparación con el control y con el tratamiento de 5 rotíferos $\mathrm{mL}^{-1}$ (Tabla 1 ).
La concentración de fosfatos fue estadísticamente similar en todos los tratamientos. La concentración de compuestos nitrogenados aumentó en función de la concentración de rotíferos, presentando valores significativamente más altos en los dos tratamientos con la mayor concentración (15 y 20 rotíferos $\mathrm{mL}^{-1}$ ), los resultados de la ANOVA muestran los valores de F y P de dichos análisis estadísticos (Tabla 1).

La concentración de nitratos en el control y en el tratamiento con 5 rotíferos $\mathrm{mL}^{-1}$ fue significativamente menor que en el de 20 rotíferos $\mathrm{mL}^{-1}$, mientras que no hubo diferencias entre los demás tratamientos. La concentración de nitritos fue mayor en los tratamientos con 10, 15 y 20 rotíferos $\mathrm{mL}^{-1}$. La concentración de nitrógeno amoniacal en el control fue inferior a los demás tratamientos, mientras que el valor más alto se obtuvo en el tratamiento con 20 rotíferos $\mathrm{mL}^{-1}$ (Tabla 1).

El crecimiento semanal presentó una relación directa con la concentración de rotíferos añadidos (Fig. 1). Lo mismo fue observado para los demás parámetros de producción (Tabla 2). El tratamiento con 20 rotíferos $\mathrm{mL}^{-1}$ presentó un crecimiento superior a los demás; el de 15 rotíferos $\mathrm{mL}^{-1}$ fue superior al control pero no fue diferente a los de 10 y 5 rotíferos $\mathrm{mL}^{-1}$. La tasa de crecimiento específica TCE, fue mayor en los tratamientos de $15 \mathrm{y}$ 20 rotíferos $\mathrm{mL}^{-1}$, mientras que los valores más bajos se obtuvieron en los tratamientos con 0 y 5 rotíferos $\mathrm{mL}^{-1}$ (Tabla 2).

\section{Tabla 1}

\section{Promedio y desviación estándar (DS) de parámetros de calidad del agua en los tratamientos con distintas concentraciones de rotíferos}

Mean and standard deviations of water quality parameters in the treatments with different concentrations of rotifers

\begin{tabular}{|c|c|c|c|c|c|c|c|}
\hline & Control & 5 rotíferos $\mathrm{mL}^{-1}$ & 10 rotíferos $\mathrm{mL}^{-1}$ & 15 rotíferos $\mathrm{mL}^{-1}$ & 20 rotíferos $\mathrm{mL}^{-1}$ & $\begin{array}{c}\text { Resultados } \\
\text { ANOVA } \\
\text { F }\end{array}$ & $P$ \\
\hline Temp. $\left({ }^{\circ} \mathrm{C}\right)$ & $26,64 \pm 0,19 a$ & $26,67 \pm 0,14 a$ & $26,59 \pm 0,11 a$ & $26,43 \pm 0,65 a$ & $26,68 \pm 0,13 a$ & 0,30 & 0,87 \\
\hline $\mathrm{O}_{2}\left(\mathrm{mg} \mathrm{L}^{-1}\right)$ & $7,34 \pm 0,47 \mathrm{a}$ & $7,34 \pm 0,31 \mathrm{a}$ & $7,59 \pm 0,37 \mathrm{a}$ & $7,28 \pm 0,23 \mathrm{a}$ & $7,48 \pm 0,33 \mathrm{a}$ & 0,39 & 0,81 \\
\hline Sal. $\left(\mathrm{g} \mathrm{Kg}^{-1}\right)$ & $36,33 \pm 0,33 \mathrm{a}$ & $36,80 \pm 0,10 \mathrm{a}$ & $36,65 \pm 0,52 \mathrm{a}$ & $36,55 \pm 0,79 a$ & $36,61 \pm 0,22 \mathrm{a}$ & 0,41 & 0,79 \\
\hline $\mathrm{pH}$ & $7,98 \pm 0,10 b$ & $7,95 \pm 0,85 b$ & $7,81 \pm 0,10 \mathrm{ab}$ & $7,77 \pm 0,09 \mathrm{a}$ & $7,76 \pm 0,09 a$ & 4,63 & 0,02 \\
\hline $\mathrm{PO}_{4}{ }^{3-}\left(\mathrm{mg} \mathrm{L}^{-1}\right)$ & $1,03 \pm 0,11 \mathrm{a}$ & $1,08 \pm 0,22 \mathrm{a}$ & $1,06 \pm 0,19 a$ & $1,08 \pm 0,09 a$ & $1,01 \pm 0,13 a$ & 0,13 & 0,97 \\
\hline $\mathrm{NH}_{4}^{+}$ & $0,69 \pm 0,06 a$ & $0,72 \pm 0,06 b c$ & $0,76 \pm 0,01 b c$ & $0,78 \pm 0,07 \mathrm{~cd}$ & $0,85 \pm 0,02 d$ & 4,49 & 0,002 \\
\hline $\mathrm{NO}_{2}^{-}$ & $1,80 \pm 0,56 a$ & $1,94 \pm 0,24 a$ & $2,74 \pm 0,63 b$ & $3,18 \pm 0,21 b$ & $3,38 \pm 0,49 b$ & 7,41 & 0,005 \\
\hline $\mathrm{NO}_{3}^{-}$ & $0,43 \pm 0,08 \mathrm{a}$ & $0,45 \pm 0,03 \mathrm{a}$ & $0,47 \pm 0,08 \mathrm{ab}$ & $0,49 \pm 0,02 \mathrm{ab}$ & $0,55 \pm 0,04 b$ & 1,85 & 0,02 \\
\hline
\end{tabular}

La ANOVA paramétrica, mediante letras diferentes, indica que hay diferencias estadísticamente significativas entre tratamientos $(P<0,05)$ 
La biomasa fue mayor en el tratamiento con mayor concentración de rotíferos $\left(20 \mathrm{~mL}^{-1}\right)$ seguido de el tratamiento de 15 rotíferos $\mathrm{mL}^{-1}$. No se encontraron diferencias significativas entre el resto de los tratamientos (Tabla 2).

La concentración de rotíferos tuvo un efecto positivo en la supervivencia de los camarones. La mayor supervivencia se obtuvo en el tratamiento con 20 rotíferos $\mathrm{mL}^{-1} \mathrm{y}$ la menor se observó en el tratamiento control.

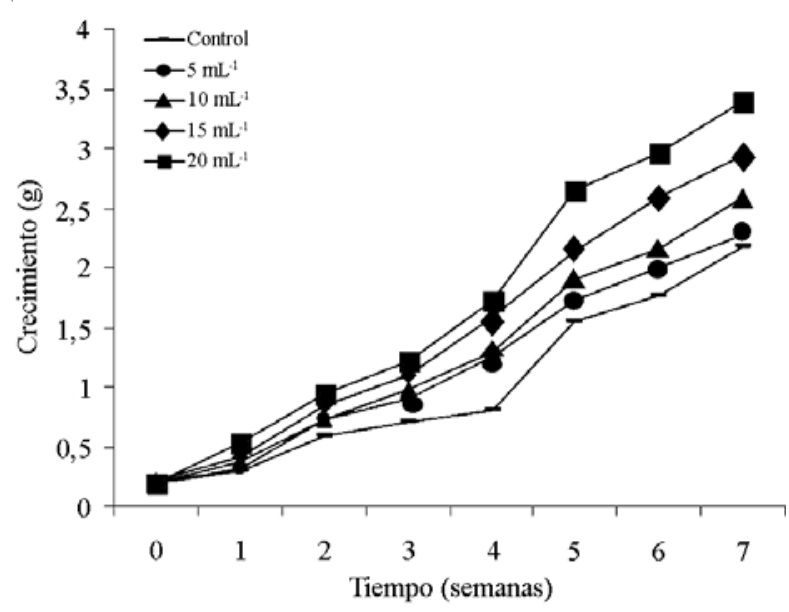

Figura 1

Crecimiento semanal de los camarones en los 5 tratamientos

Weekly growth of shrimp in 5 treatments
El FCA fue significativamente menor a medida que aumentaba el la concentración de rotíferos (Tabla 2).

\section{Discusión}

El alimento natural juega un rol crucial en las primeras etapas de vida de los camarones peneidos; sin embargo, se ha inferido sobre los beneficios que podría aportar en la engorda de esos crustáceos (Molina-Poveda et al. 2006).

Respecto a las condiciones ambientales prevalecientes durante el desarrollo del experimento, la temperatura fue similar a la reportada en verano para la región y al igual que el oxígeno, la salinidad y el $\mathrm{pH}$, se mantuvieron en el intervalo recomendado para el cultivo de camarón blanco (Martínez-Córdova 1999). La salinidad registrada en los cinco tratamientos fue similar a la óptima sugerida por Lee \& Wickins (1992) para el cultivo de L. vannamei. Aunque el pH presentó valores bajos, sobre todo en los tratamientos con altas concentraciones de rotíferos, se encontró dentro del intervalo recomendado por Lee \& Wickins (1992) y Clifford (1994). Estos bajos valores de $\mathrm{pH}$ se debieron a la alta densidad de camarones y rotíferos, que generaron metabolitos ácidos como el amonio. El oxígeno disuelto se mantuvo por encima de 5 $\mathrm{mg} \mathrm{L}^{-1}$ en todos los tratamientos, lo cual ha sido sugerido como óptimo por diversos autores (Lee \& Wickins 1992, Clifford 1994).

Los fosfatos y nitratos, se encontraron dentro del rango recomendado para los estanques camaronícolas (Teichert-Coddington 1994).

Tabla 2

Promedio y desviación estándar (DS) de los parámetros de producción de camarones (L. vannamei) alimentados con diferentes concentraciones de rotíferos

Mean and standard deviation of the production parameters of shrimp (L. vannamei) fed with differents rotifer concentrations

\begin{tabular}{cllcll}
\hline Tratamiento & Talla final $(\mathrm{g})$ & Biomasa $(\mathrm{g})$ & Supervivencia (\%) & FCA & TCE (g) \\
\hline Control & $2,18 \pm 0,31 \mathrm{a}$ & $29,92 \pm 5,36 \mathrm{a}$ & $62,12 \pm 2,62 \mathrm{a}$ & $2,74 \pm 0,09 \mathrm{~b}$ & $0,044 \pm 1,9 \cdot 10^{-5} \mathrm{a}$ \\
5 rotíferos $\mathrm{mL}^{-1}$ & $2,26 \pm 0,31 \mathrm{a}$ & $34,81 \pm 5,10 \mathrm{ab}$ & $69,70 \pm 2,62 \mathrm{~b}$ & $2,73 \pm 0,14 \mathrm{~b}$ & $0,046 \pm 3,7 \cdot 10^{-5} \mathrm{ab}$ \\
10 rotíferos $\mathrm{mL}^{-1}$ & $2,59 \pm 0,34 \mathrm{ab}$ & $40,39 \pm 3,74 \mathrm{~b}$ & $71,21 \pm 6,94 \mathrm{~b}$ & $2,66 \pm 0,24 \mathrm{~b}$ & $0,048 \pm 1,6 \cdot 10^{-5} \mathrm{ab}$ \\
15 rotíferos $\mathrm{mL}^{-1}$ & $2,95 \pm 0,28 \mathrm{bc}$ & $51,98 \pm 3,22 \mathrm{c}$ & $80,30 \pm 2,64 \mathrm{c}$ & $2,32 \pm 0,44 \mathrm{ab}$ & $0,049 \pm 1,3 \cdot 10^{-5} \mathrm{bc}$ \\
20 rotíferos $\mathrm{mL}^{-1}$ & $3,40 \pm 0,07 \mathrm{c}$ & $69,11 \pm 3,41 \mathrm{~d}$ & $92,42 \pm 2,62 \mathrm{~d}$ & $1,94 \pm 0,54 \mathrm{a}$ & $0,053 \pm 8,2 \cdot 10^{-6} \mathrm{c}$ \\
\hline
\end{tabular}

La ANOVA no paramétrica mediante letras diferentes indica que hay diferencias estadísticas entre tratamientos $(P<0,05)$ 
Las altas concentraciones de compuestos nitrogenados en los tratamientos con 10, 15 y 20 rotíferos $\mathrm{mL}^{-1}$ son producto de la descomposición de alimento no consumido y heces fecales. El amonio es además, producto de las excreciones nitrogenadas, tanto de los camarones como de los rotíferos. Estos valores coinciden con los encontrados por Martínez-Córdova et al. (2003) en experimentos con camarones cultivados a altas densidades. Buttle et al. (1995) encontraron que dietas ricas en proteínas suministradas a peces (Ictalurus) provocan que éstos eliminen mayores cantidades de desechos nitrogenados (principalmente amonio). Por otra parte, el efecto estresante de las condiciones del cultivo también pudo provocar un aumento en la excreción de amonio, tal como sucede con otros peneidos (Cuzon et al. 1980). Otra razón del incremento de amonio fue la ausencia de microalgas dentro de las unidades experimentales, ya que se ha reportado que las microalgas consumen y por lo tanto reducen la concentración de nitrógeno amoniacal (Burford et al. 2002). No obstante, con excepción del tratamiento con 20 rotíferos $\mathrm{mL}^{-1}$, los valores de nitrógeno amoniacal, se encontraron dentro de los límites de seguridad considerados para el cultivo de la especie que es de $0,8 \mathrm{mg} \mathrm{L}^{-1}$ (Xia et al. 2004). En el caso de la elevada concentración de amonio (0,85 mg $\mathrm{L}^{-1}$ ) en el tratamiento de 20 rotíferos $\mathrm{mL}^{-1}$, se considera que no hubo un efecto negativo sobre los camarones, ya que fue el tratamiento que presentó los valores más altos de supervivencia y parámetros de producción.

El incremento en los parámetros de producción del camarón debido al incremento en la concentración de rotíferos concuerda con otros reportes en donde se ha encontrado que la presencia de organismos zooplanctónicos en cantidades considerables, mejora el crecimiento y la supervivencia de peces y crustáceos, ya sea en condiciones de laboratorio o granjas comerciales (Fengqi 1996, Clifford 1994, Jory 2000, MartínezCórdova et al. 2003, 2004).

En el presente estudio, el crecimiento, la biomasa, la supervivencia, el FCA y la TCE se vieron favorecidos con las altas concentraciones de rotíferos. Es necesario puntualizar que las densidades utilizadas corresponden a un cultivo súper-intensivo y que por lo tanto los parámetros de producción no son comparables con aquellos obtenidos en sistemas semi-intensivos. Se ha demostrado que el crecimiento del camarón en cultivo está inversamente relacionado con la densidad (MartínezCórdova 1999). Sin embargo, los parámetros de producción en el tratamiento con 20 rotíferos $\mathrm{mL}^{-1}$, fueron similares o incluso superiores a los reportados en otros cultivos intensivos (Kongkeo 1997). Wyban \& Sweeney (1989) trabajaron con un sistema intensivo de camarón blanco (45 ind. $\mathrm{m}^{-2}$ ), comenzando con una talla similar a la del presente experimento $(0,27 \mathrm{~g})$ y obtuvieron un FCA por encima de 2 que es similar al obtenido en este estudio a la densidad de 15 rotíferos $\mathrm{mL}^{-1}$ pero inferior al de 20 rotíferos $\mathrm{mL}^{-1}$. Ellos utilizaron una dieta óptima con alto contenido de proteína (>40\%), lo cual representa un alimento costoso. A pesar que la concentración de organismos fue más elevada (122 ind. $\mathrm{m}^{-2}$ ) en este experimento, se igualaron e incluso superaron los parámetros de producción utilizando altas concentraciones de rotíferos.

Del presente estudio se puede concluir que el uso de los rotíferos como alimento natural exógeno, sobre todo a densidades altas, tiene efectos positivos en la respuesta productiva del camarón blanco. Aun cuando también se observaron efectos sobre algunos parámetros de la calidad del agua, especialmente $\mathrm{pH}$ y nitrógeno amoniacal, estos no fueron importantes como para ser un impedimento en el uso de estos organismos.

Los resultados demuestran que es posible mantener a los organismos en cultivos súper-intensivos y bajo techo cuando las condiciones climáticas aún no son apropiadas para la siembra, tal como sucede en países alejados de la línea ecuatorial (Martínez-Porchas 2006). De esta manera puede comenzarse el ciclo de cultivo días o semanas antes de que la temperatura ambiental alcance el punto óptimo para la engorda de los camarones.

Es indispensable realizar un estudio económico para determinar si los beneficios observados justifican los gastos operativos para el uso de este alimento natural exógeno.

\section{Agradecimientos}

Agradecemos al Centro de Desarrollo Tecnológico de Especies Marinas (CEDETEM), Tomatlán Jalisco, México por la donación de la cepa de rotíferos. A la empresa Maricultura del Pacífico S. A de C.V. ubicada al norte de Bahía de Kino, Sonora, por la donación de las postlarvas de camarón necesarias para el presente estudio. Al CONACYT por la beca otorgada para los estudios de postgrado del estudiante Alfredo Campaña Torres y a los evaluadores de la Revista Biología Marina y Oceanografía por el apoyo brindado para la publicación del presente artículo.

\section{Literatura citada}

Arnold SJ, MJ Sellars, PJ Crocos \& GJ Coman. 2006. An evaluation of stocking density on the intensive production of juvenile brown tiger shrimp (Penaeus esculentus). Aquaculture 256: 174-179. 
Ballester E, W Wasielesky, R Cavalli \& P Abreu. 2007. Nursery of the pink shrimp Penaeus paulensis in cages with artificial substrates: biofilm composition and shrimp performance. Aquaculture 269: 355-362.

Barraza-Guardado RH. 1996. Estudios de los principales componentes de la productividad natural en estanques durante la preengorda de Penaeus vannamei Boone, 1931. Tesis de Maestría, Universidad de Sonora, Hermosillo, Sonora, 124 pp.

Burford MA, NP Preston, PM Glibert \& WC Dennison. 2002. Tracing the fate of ${ }^{15} \mathrm{~N}$-enriched feed in an intensive shrimp system. Aquaculture 206: 199-216.

Buttle LG, RF Uglow \& IG Cowx. 1995. The effect of diet and photoperiod on ammonia efflux rate of the African catfish, Clarias gariepinus (Burchell, 1822). Aquaculture Research 26: 895-900.

Cabrera T, J Rosas, A Velásquez \& J Millán. 2002. Cultivo de copépodos en Venezuela. Panorama Acuícola 7: 16-19.

Castille FL \& AL Lawrence. 1986. Relationship between maturation and biochemical composition of the gonads and digestive glands of the shrimps Penaeus aztecus and Penaeus setiferus (L,). Journal of Crustacean Biology 9: 202-211.

Chiu IL \& HY Chien. 1992. Juvenile Penaeus monodon as an effective zooplankton predator. Aquaculture 103: 35-44.

Clifford HC. 1994. Semi-intensive sensation. A case study in marine shrimp pond management. World Aquaculture 25: 98-104.

Conover WJ. 1980. Practical nonparametric statistics, 493 pp. John Wiley \& Sons, New York.

Cortés-Jacinto E, H Villarreal-Colmenares, R CiveraCerecedo \& LR Martínez-Córdova. 2003. Effect of dietary protein level on growth and survival of juvenile freshwater crayfish Cherax quadricarinatus (Decapoda: Parastacidae). Aquaculture Nutrition 9: 207-213.

Cuzon G, C Cahu, JF Aldrin, JL Messager, G Sthepan \& M Mevel. 1980. Starvation effect on metabolism of Penaeus japonicus. Proceedings of the World Mariculture Society 11: 410-423.

De la Lanza EG \& PS Hernández. 1998. Nutrientes y productividad primaria en sistemas acuícolas. En: MartínezCórdova L (ed). Ecología de los sistemas acuícolas, pp. 2766. AGT Editor, México.

De Lima LCM \& LP Souza-Santos. 2007. The ingestion rate of Litopenaeus vannamei larvae as a function of Tisbe biminiensis copepod concentration. Aquaculture 271: 411419.

De Schryver P, R Crab, T Deforidt, N Boon \& W Verstraete. 2008. The basics of bio-flocs technology: The added value for aquaculture. Aquaculture 277: 125-137.

Enriquez F. 2003. Variación del bentos, con énfasis en la comunidad de poliquetos en una laguna de descarga de una granja camaronícola. Tesis de Maestría, Universidad de Sonora, Hermosillo, Sonora, 98 pp.
FAO. 2006. State of world aquaculture 2006. FAO Fisheries Technical Paper 500: 1-134.

Fengqi L. 1996. Production and application of rotifers in aquaculture. Aquaculture Magazine 22: 16-22.

Fukusho K. 1989. Biology and mass production of the rotifer, Brachionus plicatilis. International Journal of Aquaculture \& Fisheries Technology 1: 232-240.

Guillard RRL. 1975. Culture of phytoplankton for feeding marine invertebrates. En: Smith WL \& MH Chanley (eds). Culture of marine invertebrate animals, pp. 26-60. Plenum Press, New York.

Gutierrez-Venegas L. 2006. Reporte técnico-económico del cultivo de camarón en México. Industria Acuícola 2: 10-13.

Jory DE. 1996. Management of natural productivity in marine shrimp semi-intensive ponds. Aquaculture Magazine 21: 90-100.

Jory DE. 2000. General concerns for managements of biota in progress shrimp ponds. Aquaculture Magazine 26: 76-80.

Kongkeo H. 1997. Comparison of intensive shrimp farming systems in Indonesia, Philippines, Taiwan and Thailand. Aquaculture Research 28: 789-796.

Lee DOC \& JF Wickins. 1992. Crustaceans farming, 392 pp. Halsted Press/Wiley, New York.

Martínez Córdova LR. 1999. Cultivo de camarones peneidos, principios y prácticas, 298 pp. AGT Editor, México.

Martinez-Cordova LR \& LF Enriquez-Ocaña. 2007. Study of benthic fauna in the discharge lagoon of a shrimp farm with special emphasis on the polychaetes. Online Journal of Biological Sciences 7: 12-17.

Martínez-Córdova LR \& E Peña-Messina. 2005. Biotic communities and feeding habits of Litopenaeus vannamei (Boone 1931) and Litopenaeus stylirostris (Stimpson 1974) in monoculture and polyculture semi-intensive ponds. Aquaculture Research 36: 1075-1084.

Martínez-Córdova L, N Pasten \& R Barraza. 1998. Effect of fertilization on growth survival, food conversion ratio and production of Pacific white shrimp Penaeus vannamei in earthen ponds in Sonora, Mexico. The Progressive Fish Culturist 60: 101-108.

Martínez-Córdova L, A Campaña-Torres \& M PorchasCornejo. 2002. Promotion and contribution of biota in low water exchange ponds farming blue shrimp, Litopenaeus stylirotris (Stimpson). Aquaculture Research 33: 27-32.

Martínez-Córdova L, A Campaña-Torres \& M PorchasCornejo. 2003. Dietary protein level and food management in the culture of blue shrimp (Litopenaeus stylirostris) and white shrimp (L. vannamei) in microcosms. Aquaculture Nutrition 9: 155-160.

Martínez-Córdova LR, A Campaña-Torres \& M Martínez Porchas. 2004. Manejo de la productividad natural en el cultivo del camarón. En: Cruz-Suárez LE, D Ricque-Marie, MG Nieto-López, D Villarreal, U Scholz \& M González. Avances en nutrición acuícola VII. Memorias del VII Simposium Internacional de Nutrición Acuícola. 16-19 Noviembre, 2004. Hermosillo, Sonora, pp. 671-694. 
Martínez-Porchas M. 2006. Efecto de la proporción proteína: energía dietética en el desempeño biológico de Litopenaeus vannamei en baja temperatura. Tesis de Maestría, Universidad de Sonora, Hermosillo, Sonora, 47 pp.

McKinnon AD, S Duggan, PD Nichols, MA Rimmer, G Semmens \& B Robino. 2003. The potential of tropical paracalanid copepods as live feeds in aquaculture. Aquaculture 223: 89-106.

Molina-Poveda C, LR Martínez-Córdova \& W QuadrosSeiffert. 2006. Alimentación y manejo de la productividad natural. En: Rosas C, O Carrillo, R Wilson \& E Andreata (eds). Estado actual y perspectivas de la nutrición de los camarones peneidos cultivados en Iberoamérica, pp. 229274. CYTED, México.

Palmtag MR, CK Faulk \& GJ Holt. 2006. Highly unsaturated fatty acid composition of rotifers (Brachionus plicatilis) and Artemia fed various enrichments. Journal of the World Aquaculture Society 37: 126-131.

Peña-Mesina E. 1999. Comparación del consumo de alimenticio de Litopenaeus vannamei y Litopenaeus stylirostris durante la engorda, bajo condiciones de bicultivo y monocultivo semi-intensivos, en estanques de bajo recambio. Tesis de Maestría, Universidad de Sonora, Hermosillo, Sonora, 91 pp.

Pontes CS, MF Arruda, A de Lara-Menezes, A Pereira de Lima \& P Pereira de Lima. 2006. Daily activity pattern of the marine shrimp Litopenaeus vannamei (Boone 1931) juveniles under laboratory conditions. Aquaculture Research 37: 1001-1006.

Sokal RR \& F J Rohlf. 1979. Biometría. Principios y métodos estadísticos en la investigación biológica, 832 pp. H. Blume Ediciones, Barcelona.
Tacon AGJ. 2002. Global review of feeds and feed management practices in shrimp aquaculture, $68 \mathrm{pp}$. Report prepared under the world bank, NACA, WWF and FAO Consortium program on shrimp farming and the environment. Work in Progress for Public Discussion. [en línea] <www.enaca.org/shrimp>

Teichert-Coddington D. 1994. La calidad del agua y su manejo en estanques de camarón. En: Zendejas-Hernández J (ed) Memorias del Seminario Internacional de Camaronicultura, Camaron 94. Mazatlán, Sinaloa, México, pp. 12-29.

White D. 1986. Biological principles of pond culture: Sediment and benthos. En: Lannan E, RO Smitherman \& G Tchobanoglous (eds). Principles and practices of pond aquaculture, pp. 15-19. Oregon State University Press, Corvallis.

Wilcox JA, PL Tracy \& NH Marcus. 2006. Improving live feeds: Effect of a mixed diet of copepod nauplii (Acartia tonsa) and rotifers on the survival and growth of firstfeeding larvae of the southern flounder, Paralichthys lethostigma. Journal of the World Aquaculture Society 37: 113-120.

Wyban JA \& JN Sweeney. 1989. Intensive shrimp growth trials in a round pond. Aquaculture 76: 215-225.

Xia LZ, LZ Yang \& MC Yan. 2004. Nitrogen and phosphorus cycling in shrimp ponds and the measures for sustainable management. Environmental Geochemistry and Health 26: 245-251.

Zendejas-Hernández J. 1994. La camaronicultura en México. Memorias del Seminario Internacional de Camaronicultura, Camarón 94', Mazatlán, Sinaloa, México, pp. 1-12.

Recibido el 30 de octubre de 2008 y aceptado el 9 de abril de 2009 